Beata Brzozowska-Zburzyńska

Uniwersytet Marii Curie-Skłodowskiej w Lublinie beata.brzozowska-zburzynska@poczta.umcs.lublin.pl

\title{
Análisis contrastivo de los elementos constitutivos de las expresiones temporales de base nominal en español y en polaco
}

(función de localización simultánea)

\begin{abstract}
Resumen:
El objetivo de esta investigación es analizar las estructuras de las expresiones temporales que se emplean en español y polaco para desempeñar la función sintáctica de complemento circunstancial de tiempo. Siguiendo a Wilk-Racięska, creemos que cualquier elemento lingüístico, tanto léxico como gramatical, refleja la visión del mundo que comparten los hablantes de una misma lengua. Proponemos, por lo tanto, un estudio contrastivo en el cual se presentarán estructuras más o menos complejas que incluirán, necesariamente, un sustantivo acompañado de elementos facultativos, tales como preposiciones, artículos, adjetivos, marcas casuales, etc. Queremos demostrar que la aparición de estos elementos lingüísticos no es accidental y se puede explicar tomando en cuenta aspectos semánticos, pragmáticos y cognitivos propios de cada una de las lenguas estudiadas.
\end{abstract}

Palabras clave: expresiones temporales, estructuras, visión del mundo

\begin{abstract}
:
Comparative Analysis of the Constitutive Elements Temporal Expressions with a Nominal Base in Spanish and Polish (Simultaneous Function)

The aim of the study is to analyze the structures of temporal expressions used in Spanish and Polish which assume the syntactic function of circumstantial
\end{abstract}


complement of time. Following Wilk-Racięska, we believe that any linguistic element, both lexical and grammatical, reflects the vision of the world shared by speakers of the same language. We propose, therefore, a contrastive study in which more or less complex structures will be presented, which will obligatorily include a noun accompanied by optional elements such as prepositions, articles, adjectives, casual markers and others. We want to demonstrate that the appearance of these linguistic elements is not accidental and can be explained by taking into account semantic, pragmatic and cognitive aspects specific to each of the languages studied.

Keywords: temporary expressions, structures, vision of the word

El objetivo de este trabajo es presentar los elementos lingüísticos que forman parte de una expresión temporal de base nominal en español y en polaco ${ }^{1}$. Las expresiones con estas características, que a nivel sintáctico cumplen la función de complemento circunstancial, son sintagmas nominales o preposicionales, más o menos complejos, con un solo elemento obligatorio, que es el sustantivo. Hablaremos, por lo tanto, de un subgrupo de elementos lingüísticos que sirven para ubicar las acciones verbales en el tiempo junto con otros subgrupos que desempeñan la misma función, de entre los cuales destacan los adverbios de tiempo como elementos prototípicos. Sin embargo, como observa Carbonero Cano:

(...) por evidentes razones de economía del lenguaje, el sistema temporal no dispone de una unidad adverbial mínima de tipo léxico, para cada situación temporal que haya que expresar. Por ejemplo, tomando la unidad «noche», para el pasado tenemos una unidad léxica: anoche; y, sin embargo, para el presente o futuro necesitaremos otros recursos, construcciones complejas de nivel sintagma: esta noche (presente), mañana noche o la próxima noche (futuro). La lengua no dispone de unidades léxicas para tales ocasiones. (...) Esa es la razón por la que encontramos que en el cuadro

${ }^{1}$ En el artículo presentamos algunos resultados de una investigación mucho más extensa que se publicó en un volumen monográfíco (BrzozowskaZburzyńska, 2017). 
general del sistema adverbial hay algunas «casillas vacías» cuya noción no dispone de unidad específica y habrá de ser llenada por otra unidad más compleja que la exprese (Carbonero Cano, 1979: 115).

En este trabajo revisaremos las principales estructuras que, en español y en polaco, se pueden formar, para realizar la función circunstancial, con sustantivos, principalmente temporales. Como explica Martínez (1995: 129), podemos hablar de los sustantivos temporales de cuantificación imprecisa (tiempo, momento, época, instante), y de los de cuantificación precisa (segundo, hora, día, tarde, lunes, mes, siglo, etc.). Además de estos, hay también sustantivos que nombran eventos o actividades y en los que el sema temporal no es principal sino periférico, tales como: reunión, vacaciones, comida, lectura (José, 2003: 31), que también pueden formar núcleos de las expresiones temporales que nos interesan. No obstante, en este artículo nos limitaremos al análisis de los sustantivos temporales de cuantificación precisa, por lo que tampoco nos referiremos al grupo de sustantivos que designan partes de cualquier intervalo temporal, es decir: principio, final, caída, mitad, etc. (Borillo, 1998: 133).

Cuando estudiamos y comparamos lenguas distintas, podemos constatar que se diferencian tanto a nivel léxico como gramatical. Por eso, también podemos observar que tanto el lexicón como la gramática tienen como objetivo ordenar y simbolizar el contenido conceptual de lo comunicado. Su carácter es "imaginístico"2 (Langacker, 1998: 45). Eso quiere decir que cualquier elemento lingüístico, sea cual sea su estatus (elemento léxico, elemento gramatical) y su extensión (un simple morfema, una palabra o una estructura compleja), refleja el modo de ver y entender el mundo que comparten los hablantes de una misma lengua, esto es, su visión del mundo ${ }^{3}$.

${ }^{2}$ El adjetivo imaginístico es un neologismo que se suele emplear en los trabajos de los lingüistas que se dedican al paradigma cognitivista, sobre todo, formando sintagmas nominales de tipo: esquemas imaginísticos, carácter imaginístico de la representación lingüística, etc.

3 "La visión del mundo es un concepto complejo que englobaría modelos cognitivos convencionales, valores, emociones, escenarios sociales, situaciones, 
Cuando comparamos las visiones del mundo de los hablantes de lenguas diferentes, podemos observar que las diferencias son menores cuando nos referimos a las lenguas que pertenecen a la misma cultura macro ${ }^{4}$ y mayores cuando hablamos de culturas no emparentadas, pero siempre las diferencias siempre se dan, incluso cuando hablamos de lenguas pertenecientes a la misma familia genealógica, o, como en el caso del castellano, de sus variedades geográficas 5 .

El español y el polaco son dos lenguas europeas que se han desarrollado en el marco de la misma cultura macro, por lo que comparten muchas ideas sobre la organización y el modo de entender el mundo, así como comportamientos culturales y sociales y, por consiguiente, lingüísticos. Sin embargo, presentan también muchas diferencias, tanto a nivel léxico como gramatical, que se deben a distintos factores tanto intra como extralingüísticos, y sobre todo reflejan otra imaginería ${ }^{6}$.

Para este estudio contrastivo hemos elegido expresiones pertenecientes al dominio del tiempo ya que, como asegura Guriewicz (1976: 95): "Hay pocos elementos que caracterizan tan bien la esencia de una cultura como el modo de entender el tiempo"'.

estados de ánimo, esquemas mentales metafóricos y metonímicos, en definitiva, toda una configuración cultural y ética a través de la cual evaluamos o asumimos ciertos comportamientos, eventos y realidades" (Luque Durán, 2004: 491, véase también Wilk-Racięska, 2007, 2009).

${ }^{4}$ Una cultura macro puede agrupar a varias culturas de menor alcance y se caracteriza por compartir los mismos principios filosóficos, religiosos, sociales, culturales, etc. (p. ej. cultura macro europea versus cultura macro asiática) (Wilk-Racięska, 2007: 446).

${ }^{5}$ Cuando hablamos del castellano, no podemos olvidar el hecho de que esta lengua se ha desarrollado en lugares del mundo muy diferentes, por lo que podemos encontrar elementos lingüísticos que reflejan tanto la visión del mundo europea, como las de otras culturas macro muy diferentes (por ejemplo, la andina). Para ver algunas particularidades del español europeo contrastado con el de América Latina, se recomienda leer el libro de Wilk-Racięska (2012).

${ }^{6}$ Según Palmer (2000: 24) es el modo de ver y entender el mundo gracias a la imaginación y facultades mentales de los hablantes.

${ }^{7}$ Traducción nuestra. 
La concepción del tiempo para los hablantes españoles y polacos la podemos apreciar analizando las ideas generales sobre él que comparten los hablantes de estas dos lenguas ${ }^{8} ; \mathrm{y}$ también, al considerar las expresiones lingüísticas concretas que se refieren al tiempo, donde podemos observar tanto las coincidencias como las divergencias en el modo de dividirlo en entidades menores y también en el modo de conceptualizar estas entidades como puntos, duraciones o estructuras complejas.

Las expresiones temporales cuya base es un sustantivo y que desempeñan la función de complemento circunstancial de tiempo suelen aparecer, tanto en español como en polaco, precedidas de una preposición. Martínez (1995: 129), siguiendo las ideas ya clásicas de Alarcos Llorach, explica que las preposiciones desempeñan la función de elementos traspositores a la categoría adverbial de otros elementos lingüísticos, tales como sustantivos, adjetivos y otros, es decir, que participan en el proceso de adverbialización. Sin embargo, existen también expresiones con lo que Haspelmath (1997: 116) llama el marcador cero, es decir, que no llevan ninguna preposición, pero funcionan como CCT. Suelen ser sustantivos modificados por determinantes, adjetivos o complementos, aunque también haya estructuras unimembres. Además de estas, existen igualmente estructuras que el lingüista alemán llama minimal marked, es decir, construcciones sin preposición, pero con una marca casual concreta. Aquí aparece la primera gran diferencia entre las dos lenguas que estudiamos. Desde el punto de vista tipológico el español es una lengua de tipo: 'tiempo + artículo' y el polaco es una lengua de tipo: 'aspecto + caso' (Nowikow, 2013: 66). Por eso, en la estructura de las expresiones temporales españolas aparecerá el artículo definido como uno de los elementos constitutivos facultativos, mientras que en polaco serán las marcas morfológicas de los casos de la declinación las que influirán

${ }^{8}$ Algunas ideas generales sobre el tiempo que vienen de la antigua filosofía y de la física, que comparten los hablantes de las lenguas europeas, se pueden consultar en el artículo de Wilk-Racięska (2012b). 
en la interpretación semántica de las expresiones temporales en las que aparecen.

El hecho de que encontremos estructuras preposicionales, así como otras que no contienen preposiciones, está relacionado con tres maneras principales de percibir habitualmente el tiempo (Honeste, 1997: 163) en la cultura macro europea:

- el tiempo no dimensionado ni configurado, percibido de modo global (es el caso de las construcciones gramaticales sin preposiciones);

- el tiempo dimensionado en duración (alternancias entre las estructuras con y sin preposiciones);

- el tiempo configurado (como contenedor o como estructura compleja), en cuyo caso la presencia de las preposiciones es obligatoria. Según Haspelmath (1997), las expresiones temporales de base nominal pueden desempeñar varias funciones semánticas. Entre las más importantes el investigador alemán destaca las siguientes: localización simultánea, localización secuencial (anterior y posterior), localización secuencial durativa (anterior y posterior), distancia temporal, extensión temporal (atélica y télica), y distancia posterior.

En este estudio solamente analizaremos ejemplos de expresiones que realizan la función de localización simultánea, es decir, las que permiten localizar una acción verbal en relación a otra acción o a "un periodo de tiempo canónico" (Haspelmath, 1997: 29) de tal modo que ambos elementos de la relación, que así se establece, sean simultáneos.

A continuación, presentamos el cuadro que contiene las principales construcciones con sustantivos que designan los principales periodos canónicos del tiempo que podemos encontrar en español y polaco para la función de localización simultánea. Debemos precisar que en los grupos de sustantivos que designan las unidades principales del

${ }^{9}$ Haspelmath llama así los principales periodos en los que se puede dividir el tiempo en una cultura. Distingue tres subgrupos de estos periodos canónicos: 1) las unidades del tiempo (hora, día, mes, año); 2) las unidades principales del calendario (enero, jueves); 3) periodos cualitativos (primavera, tarde) (Haspelmath, 1997: 26). 
tiempo y del calendario, el número de estas y su estatus funcional son idénticos en español y en polaco ${ }^{10}$. No obstante, en el grupo de los sustantivos que designan periodos cualitativos, sí podemos observar algunas diferencias en cuanto al número de entidades que se pueden distinguir en ambas lenguas y su funcionamiento lingüístico ${ }^{11}$. Esto se debe a varios factores extralingüísticos propios de cada una de las dos lenguas que analizamos.

\begin{tabular}{|l|l|l|}
\hline $\begin{array}{l}\text { Entidades } \\
\text { temporales }\end{array}$ & Expresiones españolas & Expresiones polacas \\
\hline Horas & a las cuatro (horas) & o czwartej (godzinie) \\
\hline Partes del día & $\begin{array}{l}\text { durante el día/de día, } \\
\text { de noche/por la noche, } \\
\text { a mediodía, } \\
\text { a medianoche, } \\
\text { por la mañana, } \\
\text { por la tarde, } \\
\text { de madrugada }\end{array}$ & $\begin{array}{l}\text { w dzień/za dnia/dniem, } \\
\text { w nocy/nocą, } \\
\text { w południe, } \\
\text { o północy, } \\
\text { rano, } \\
\text { wieczorem, } \\
\text { nad ranem }\end{array}$ \\
\hline
\end{tabular}

${ }^{10}$ Estos sustantivos funcionan como las llamadas nomenclaturas (en la semántica estructuralista, véase, por ejemplo, la distinción entre terminologías y lenguaje primario de Coseriu de 1964), es decir son nombres que "[...] no pertenecen al lenguaje ni a las estructuraciones léxicas del mismo modo que las palabras usuales, sino que constituyen utilizaciones del lenguaje para clasificaciones diferentes de la realidad o de ciertas secciones de la realidad, al no estar estructuradas del mismo modo, al no seguir las normas del lenguaje sino las de las ciencias, las de las técnicas y las de la realidad de las cosas, por tanto es un léxico, más bien, ordenado que no nos da ningún dato de información de las cosas sólo las nombra" (Izquierdo Guzmán, 1992: 17-18). Sin embargo, según la semántica cognitiva, no hay entidades lingüísticas que no pueden recibir una interpretación semántica. Lo demuestran Cuenca \& Hilferty (1999: 71) en su definición de la palabra martes.

${ }^{11}$ A este propósito se pueden consultar otros artículos nuestros sobre los sustantivos que designan partes de un día (Brzozowska-Zburzyńska, 2012a) y otras entidades temporales (Brzozowska-Zburzyńska, 2012b). 


\begin{tabular}{|c|c|c|}
\hline Días & $\begin{array}{l}\text { el lunes, } \\
\text { este martes, } \\
\text { el viernes pasado, este/ese/ } \\
\text { aquel día }\end{array}$ & $\begin{array}{l}\text { w poniedziałek, } \\
\text { w ten wtorek, } \\
\text { w zeszły piątek, } \\
\text { w tym/tamtym dniu, } \\
\text { w ten/tamten dzień, } \\
\text { tego/tamtego dnia }\end{array}$ \\
\hline Meses & $\begin{array}{l}\text { en abril, } \\
\text { en/durante el mes de } \\
\text { mayo, } \\
\text { el próximo mes }\end{array}$ & $\begin{array}{l}\text { w kwietniu, } \\
\text { w miesiącu maju, } \\
\text { w przyszłym miesiącu }\end{array}$ \\
\hline Estaciones & $\begin{array}{l}\text { en (la) primavera, } \\
\text { en verano, } \\
\text { en otoño, } \\
\text { en invierno }\end{array}$ & $\begin{array}{l}\text { na wiosnę/wiosną, } \\
\text { w lecie/latem, } \\
\text { na jesieni/jesienią, } \\
\text { w zimie/zimą }\end{array}$ \\
\hline Años & $\begin{array}{l}\text { en } 2016, \\
\text { (durante) este/ese/aquel } \\
\text { año, } \\
\text { el próximo año, } \\
\text { el año pasado }\end{array}$ & $\begin{array}{l}\text { w } 2016 \text { roku, } \\
\text { w tym roku, } \\
\text { w przyszłym roku, } \\
\text { w zeszłym/tamtym roku }\end{array}$ \\
\hline
\end{tabular}

Como podemos apreciar al analizar las estructuras de las expresiones presentadas en el cuadro, muchas de estas aparecen introducidas por una preposición. Tanto en español como en polaco las principales preposiciones que marcan las relaciones temporales tienen un significado original espacial ${ }^{12}$. Por eso, sus significados temporales son resultado de una transposición metafórica desde el dominio espacial hacia el dominio temporal. Por lo tanto, podemos observar analogías entre la estructura conceptual de ambos dominios que consiste en una sustitución conceptual (Przybylska, 2001: 260):

- Al trajector espacial, que es un "ente espacial", le corresponde un trajector temporal, es decir, un "acontecimiento";

- Al landmark espacial, que es también un "ente espacial", le corresponde un landmark temporal, es decir, un "periodo de tiempo";

${ }^{12}$ La excepción la constituye la preposición española durante, que carece de empleos no temporales. 
- A la localización de un objeto en el espacio le corresponde la localización de un acontecimiento en el tiempo (Przybylska, 2002: 231).

Lo que nos parece interesante es que casi todos los sustantivos polacos que designan periodos canónicos de tiempo aparecen en una construcción prepositiva. La excepción la constituyen el sustantivo rano (la mañana) y el sustantivo wieczór ${ }^{13}$ (la tarde). Los demás sustantivos polacos pueden formar expresiones con preposición ${ }^{14}$. La más frecuente es la preposición $w$, que en el dominio espacial marca la interioridad. Se emplea con algunas partes del día (południe, noc, $d z i e n^{15}$ ), con los nombres de los días de la semana, con los que designan meses, años y las estaciones más prototípicas (verano e invierno). Todas estas entidades temporales (que funcionan como landmark de una relación temporal) se conciben como contenedores unidimensionales, esto es, segmentos temporales lineales limitados por un momento inicial y otro final (Przybylska, 2002: 232). Los acontecimientos marcados por el predicado de una oración en la cual aparece una expresión temporal de estas características (que funcionan como trajector de la relación temporal) se conciben como acontecimientos puntuales que ocupan la región interior del landmark (Przybylska, 2002: 232), esto es, como simultáneos.

En español, el marcador que indica la interioridad es la preposición en, que también sirve para construir expresiones temporales con la función de localización simultánea. Se emplea con los nombres de todas las estaciones del año, con el año determinado por un numeral

${ }^{13}$ Existe sin embargo la construcción pod wieczór, pero ya no es muy frecuente en el polaco actual. Se entiende como un periodo inmediatamente anterior a la caída del sol aunque muy impreciso. Se puede emplear con más facilidad en verano, cuando oscurece más tarde. La preposición pod designa, en el dominio espacial, una posición inferior en el eje vertical. Gracias a los procedimientos metafóricos se puede utilizar también en el dominio temporal (BrzozowskaZburzyńska, 2012a: 63).

${ }^{14}$ Aunque algunas alternan con construcciones sin preposición: w lecie/latem, w tym dniu/tego dnia, etc.

${ }^{15}$ Cuando este sustantivo designa parte del día de 24 horas y no una unidad del tiempo cronológico. 
(por ejemplo: en 2017), con los nombres de los meses. No aparece, sin embargo, con los nombres de los días de la semana, ni con los que designan partes de un día. Eso quiere decir que estas últimas unidades temporales no se conciben en español como contenedores cerrados.

Otras preposiciones que sirven para formar expresiones temporales de base nominal con función de localización simultánea son:

- En polaco, la preposición $o$, que sirve para marcar un punto temporal (con el sustantivo pótnoc 'medianoche' o con las horas); la preposición $z a$, que, en el caso de los nombres que indican periodos canónicos de tiempo, solamente aparece con el sustantivo $d z i e n$ ' día' y forma una expresión ya poco empleada ( $z a d n i a)$, que corresponde a la expresión española de día; la preposición $n a$, que se emplea únicamente con los nombres de las estaciones del año menos prototípicas: wiosna 'primavera', jesien' 'otoño' ${ }^{16}$; las preposiciones $\mathrm{nad} / \mathrm{pod}^{17}$, que se emplean con los sustantivos rano y wieczór, respectivamente, y que sirven para indicar de un modo poco preciso un periodo inmediatamente anterior al indicado por el sustantivo.

- En español, la preposición $a$, que marca un punto temporal con las horas, con los sustantivos mediodía ${ }^{18}$ y medianoche ${ }^{19}$; la preposición de, que aparece en las expresiones de día, de noche, de

${ }^{16}$ A propósito de este tipo de construcciones, recomendamos el artículo de Przybylska (2006: 50), donde la autora describe de un modo detallado el funcionamiento de las construcciones: na wiosnę, na jesieni. La particularidad que tienen estas no consiste solamente en el hecho de que se construyen con la preposición $n a$ y no $w$, como los nombres que designan el verano y el invierno, sino también al hecho de que el sustantivo wiosna aparece en acusativo y el sustantivo jesień en locativo.

${ }^{17}$ Son los únicos casos, en polaco, de marcadores de espacio (marcan relaciones en el eje vertical) que se emplean también para marcar relaciones temporales.

${ }^{18}$ Lo interesante es que en polaco el sustantivo poludnie (mediodía) no se concibe como puntual sino como un contenedor, pues se emplea con la preposición w y no $o$.

${ }^{19}$ Así como con otros sustantivos que marcan una parte del día más específica que, sin embargo, no hemos incluido en el cuadro, como: al amanecer, al atardecer, etc. 
madrugada ${ }^{20}$; la preposición por, que se emplea con algunos nombres de partes del día y que designa una relación topológica entre un conjunto cerrado compacto y los puntos que lo forman (López García, 2005: 226); y la preposición durante, que es el exponente natural de la discontinuidad.

En cuanto a las construcciones sin preposiciones podemos distinguir entre las unimembres, las bimembres y las complejas.

En español, las construcciones unimembres son casi inexistentes. Los únicos ejemplos que hemos encontrado son los nombres de los días de la semana, que pueden aparecer solos en enumeraciones, como: Lunes y martes no habrá clases; o cuando el nombre de un día de la semana aparece como modificador en estructuras complejas: Fue al día siguiente, lunes, por la tarde (...).

En polaco, no podemos hablar de estructuras completamente unimembres porque los sustantivos llevan siempre alguna marca casual. En esta lengua existen tres construcciones sin preposición que pueden desempañar la función de CCT: el genitivo temporal (p.ej., tego dnia), el acusativo temporal (p.ej., cały dzień) y el instrumental (p.ej., późnym popołudniem). Pero solamente la estructura con el instrumental puede ser unimembre y solo es posible con los nombres de las estaciones del año, así como con los nombres que indican partes de un día de 24 horas (latem, wieczorem) (Menzel, 2005).

Las construcciones bimembres, tanto en español como en polaco, se forman con la ayuda de algunos adjetivos determinativos (demostrativos o indefinidos).

Langacker (1991: 102) postula que los demostrativos, así como los determinantes definidos e indefinidos, son elementos que forman parte de un grupo de expresiones especiales que el lingüista norteamericano llama grounding predications, es decir, predicaciones de

${ }^{20}$ Según Brea (1985: 161), la preposición de suele indicar, en todas las lenguas románicas, la procedencia y, por extensión, también la posesión. Eso explica porque esta preposición solamente se emplea con los sustantivos que pertenecen al grupo de los periodos cualitativos (según la nomenclatura de Haspelmath); la palabra día designa aquí el periodo de tiempo caracterizado por la presencia de luz solar. 
anclaje. Lo que hacen estas expresiones es relacionar los entes de los que se habla con el fondo de anclaje que corresponde a la propia situación de comunicación y sus componentes.

Sin embargo, si comparamos las dos lenguas que son objeto de nuestro estudio, podemos constatar que solamente en español son posibles las construcciones con el artículo definido, el cual aparece en construcciones bimembres con los nombres de los días de la semana. La expresión temporal así formada designa un día de la semana concreto, pero al mismo tiempo lo determina deícticamente en referencia al momento del habla. Con los nombres que designan otras entidades temporales, el artículo puede aparecer, pero modificado por otros elementos facultativos tales como adjetivos temporales o complementos: el día dos de junio, el año pasado, la semana que viene, etc. ${ }^{21}$ Es así porque con estas otras entidades la identificación del referente temporal no es tan evidente y el hablante necesita más datos para orientarse adecuadamente en el proceso de búsqueda de las entidades correspondientes, es decir, estas entidades son menos accesibles al hablante que las que designan los días de la semana.

(...) todas las lenguas naturales disponen de mecanismos lingüísticos para que el hablante pueda marcar como más o menos accesibles aquellas entidades mentales que deberían estar cognitivamente activadas durante el proceso de comprensión (Figueras, 2002: 54).

Las representaciones mentales de los referentes inmediatamente accesibles al destinatario requieren menos informaciones descriptivas que las menos accesibles ${ }^{22}$.

En un espacio tan limitado como es el de un artículo, no hemos podido describir exhaustivamente todos los elementos constitutivos de las expresiones temporales de base nominal en español y en polaco, pero hemos intentado dar cuenta de algunas particularidades que

${ }^{21}$ En polaco, en estos contextos aparece la preposición $w$ : w zeszlym roku, w przyszłym tygodniu; o bien la construcción bimembre con el genitivo temporal: drugiego czerwca.

${ }^{22}$ Teoría de la accesibilidad de Ariel (1990, 1988). 
están relacionadas con estos elementos. Como conclusión proponemos las siguientes constataciones:

- Los sustantivos temporales dan cuenta de la división convencional del tiempo en las entidades que se distinguen en una comunidad lingüística concreta. Si comparamos el español y el polaco, podemos observar que las diferencias más importantes tienen que ver con los nombres de los periodos cualitativos, es decir, de las estaciones del año y de las partes del día. Es así porque la división del día y del año se lleva a cabo siguiendo distintas motivaciones de carácter extralingüístico, o como dice Nowikow (2005), a partir de entidades de cultura lingüisticamente operacionale ${ }^{23}$ que son dispares.

- Las preposiciones dan cuenta de la configuración conceptual de las entidades temporales como contenedores, como estructuras complejas o como duración. En el caso de la función de localización simultánea, hemos podido comprobar que el español ofrece más posibilidades de configuración que el polaco. La mayoría de los periodos temporales se conciben en esta última lengua como contenedores cerrados ${ }^{24} \mathrm{y}$ se emplean con la preposición $w$, que es el exponente de la relación de interioridad. En español, este modo de concebir los periodos temporales también aparece, pero, además, podemos observar otras configuraciones, que se manifiestan con la ayuda de otros marcadores preposicionales como por, de, durante.

- Los determinantes son elementos lingüísticos que relacionan los entes de los que se habla con el fondo de anclaje que corresponde a la propia situación de comunicación y sus componentes. Para eso sirven los adjetivos determinativos e indefinidos y también el artículo. Este último elemento permite formar expresiones temporales bimembres con los nombres de los días de la semana en

${ }^{23}$ Nowikow observa, por ejemplo, que la división del día se efectúa en polaco a partir del concepto de 'luz solar', mientras que en el español peninsular se hace a partir de las horas de las comidas (Nowikow, 2005: 412).

${ }^{24} \mathrm{La}$ excepción la constituyen los nombres de las estaciones del año no prototípicas y algunas partes del día. 
español (el martes, el jueves), lo que no es posible en polaco porque esta lengua no dispone de artículos. En polaco, los días de la semana se conciben necesariamente como contenedores.

- La complejidad de las estructuras de las expresiones temporales corresponde al nivel de su accesibilidad. Cuanto más simple es la estructura tanto mayor es el grado de accesibilidad cognitiva de las entidades temporales. Tanto en español como en polaco las estructuras unimembres son marginales ya que principalmente los nombres de las entidades temporales carecen de la autonomía semántica que permita identificar sus referentes de manera inequívoca, sin ningún tipo de modificador.

\section{Bibliografía}

ARIEL, M. (1990), Accessing Noun-Phrase Antecedents, Routledge, London-New York.

ARIEL, M. (1988), "Referring and Accessibility", Journal of Linguistics, 24, pp. 65-87.

BORILLO, A. (1998), "Les adverbes de référence temporelle comme connecteurs temporels de discours", en: Vogeleer, S., Borillo, A., Vetters, C., Vuillaume M. (eds.), Temps et discours, Peeters, Louvain-la-Neuve, pp. 131-145.

BREA, M. (1985), "Las preposiciones, del latín a las lenguas románicas", Verba, 12, pp. 147-182.

BRZOZOWSKA-ZBURZYŃSKA, B. (2017), Estudio contrastivo de las expresiones temporales de base nominal en español, francés y polaco, Wydawnictwo UMCS, Lublin.

BRZOZOWSKA-ZBURZYŃSKA, B. (2012a), “Análisis contrastivo de las expresiones temporales que indican partes del día en español, francés y polaco", Neophilologica, 24, pp. 57-65.

BRZOZOWSKA-ZBURZYŃSKA, B. (2012b), “¿Los usuarios de lenguas diferentes entienden y dividen el tiempo cronológico del mismo modo? el caso del español, francés y polaco", Studia Iberystyczne, 11, pp. 57-68, [on-line] https://doi.org/10.12797/SI.11.2012.11.05. 
CARBONERO CANO, P. (1979), Deíxis espacial y temporal en el sistema lingüístico, Universidad de Sevilla, Sevilla.

CUENCA, M. J., HILFERTY, J. (1999), Introducción a la Lingüística Cognitiva, Editorial Ariel, Barcelona.

FIGUERAS, C. (2002), "La jerarquía de accesibilidad de las expresiones referenciales en español", Revista Española de Lingüística, 32(1), pp. 53-96.

GURIEWICZ, A. (1976), Kategorie kultury średniowiecznej, Państwowy Instytut Wydawniczy, Warszawa.

HASPELMATH, M. (1997), From Space to Time. Temporal Adverbials in the World's Languages, Lincom Europa, München-Newcastle.

HONESTE, M.-L. (1997), “Approche cognitive de la syntaxe des compléments de temps sans préposition en français", Faits de langues, 5(9), pp. $155-164$.

IZQUIERDO GUZMÁN, M. L. (1992), Estudio léxico-semántico de los términos que delimitan tiempo en «día». Investigación diacrónica, [tesis doctoral], Universidad de la Laguna.

JOSÉ, L. (2003), "Noms de temps et opérations de partition: le problème de $\sim$ Il est arrivé au mois de juin de la même année $\sim$ vs $\sim *$ Il est arrivé au mois de juin de l'année ", en: Schnedecker, C., Theissen, A., Langages, 37/151, Indéfinis, définis et expression de la partition, pp. 29-42.

LANGACKER, R. W. (1998), "Wstęp do gramatyki kognitywnej”, en: Kubiński, W., Kalisz, R., Modrzejewska E. (red.), Językoznawstwo kognitywne. Wybór tekstów, Wydawnictwo Uniwersytetu Gdańskiego, Gdańsk, pp. 28-79.

LANGACKER, R. W. (1991), Foundations of Cognitive Grammar, Stanford University Press, Stanford.

LÓPEZ GARCÍA, Á. (2005), Gramática cognitiva para profesores de español L2: cómo conciben los hispanohablantes la gramática, Arco Libros, Madrid.

LUQUE DURÁN, J. (2004), Aspectos universales y particulares del léxico de las lenguas del mundo, Impredisur S.L., Granada.

MARTÍNEZ, J. A. (1995), Funciones, categorías y transposición, ISTMO, Madrid.

MENZEL, T. (2005), "O alternacji fraz przyimkowych i fraz z narzędnikiem 
w języku polskim i rosyjskim”, en: Grochowski M. (red.), Przystówki i przyimki. Studia ze sktadni i semantyki języka polskiego, Wydawnictwo Uniwersytetu Mikołaja Kopernika, Toruń, pp. 227-248.

NOWIKOW, W. (2013), "Sobre la modalización del contenido proposicional. Contraste tipológico entre lenguas románicas y eslavas", en: Pamies Bertrán A. (ed.), De lingüística, traducción y léxico-fraseología. Homenaje a Juan de Dios Luque Durán, Editorial Comares, Granada, pp. 66-72.

NOWIKOW, W. (2013), "Sobre la transmisión de la información en los contactos interculturales. Un estudio contrastivo entre el español de México y el polaco", en: Murillo Medrano J. (ed.), Actos de habla y cortesía en distintas variedades del español. Perspectivas teóricas y metodológicas. Actas del II Coloquio Internacional del Programa EDICE, Univeridad de Costa Rica/Universidad de Estocolmo, Estocolmo-Costa Rica, pp. 407-418.

PALMER, G. (2000), Lingüística cultural, Alianza Editorial, Madrid.

PRZYBYLSKA, R. (2006), "Spacjalizacja czasu w przyimkowych frazach temporalnych. Regularności i nieregularności”, en: Dąbrowska A., Nowakowska, A. (red.), Język a kultura, 19, Czas-język-kultura, Wydawnictwo Uniwersytetu Wrocławskiego, Wrocław, pp. 45-60.

PRZYBYLSKA, R. (2002), Polisemia przyimków polskich $w$ świetle semantyki kognitywnej, Universitas, Kraków.

PRZYBYLSKA, R. (2001), "Typy relacji znaczeniowych między różnymi sensami przyimka”, en: Cygal-Krupowa Z. (red.), Studia językoznawcze, Universitas, Kraków, pp. 251-262.

WILK-RACIĘSKA, J. (2012a), Entre la visión del mundo temporal y la aspectual. Casos del español sudamericano, Wydawnictwo Uniwersyetu Śląskiego, Katowice.

WILK-RACIECKA, J. (2012b), "Sobre el tiempo en filosofía, física y lingüística", Neophilologica, 24, pp. 261-269.

WILK-RACIĘSKA, J. (2009), Od wizji świata do opisu językoznawczego w kategoriach lingwistyki kulturowej. Uwagi na temat hiszpańskiej syntagmy nominalnej, Wydawnictwo Uniwersytetu Śląskiego, Katowice.

WILK-RACIECSKA, J. (2007), "Nuestro mundo, nuestras visiones del mundo y las lenguas que lo describen todo", Anuario de Estudios Filológicos, 30, pp. 439-453. 